\title{
Fabry-Perot Observations and Hydrodynamical Simulations of the Barred Spiral NGC 4123
}

\author{
Benjamin J. Weiner, J. A. Sellwood, and T. B. Williams \\ Dept. of Physics and Astronomy, Rutgers University, Piscataway NJ \\ 08855
}

\begin{abstract}
We present Fabry-Perot observations of the velocity field of gas in the barred spiral NGC 4123, and 2-D hydrodynamical simulations of the gas flow in model potentials derived from $I$-band photometry. The simulated gas flow is quite sensitive to the details of the potential, which enables us to constrain parameters such as the $M / L_{I}$ of the bar and the bar pattern speed. The observations confirm that the dust lanes along the leading edges of the bar are the locations of shocks. Requiring models to produce shocks with the correct alignment constrains the Lagrange point $L_{1}$ (corotation) to be at a radius $1.1-1.4$ times the semimajor axis of the bar, i.e. the bar is a fast rotator.
\end{abstract}

\section{Introduction}

The non-circular streaming motions in barred galaxies offer a probe of dynamical parameters such as the mass and pattern speed of the bar. Fully two-dimensional kinematic observations and models are required due to the non-axisymmetry. With observations and computational facilities now available it is possible to carry out an extensive modeling program. Fabry-Perot observations of the $\mathrm{H} \alpha$ emission line can map the velocity field of the gas with $1^{\prime \prime}-2^{\prime \prime}$ resolution. Hydrodynamical codes are necessary to calculate the gas velocities in the models, since shocks are likely to be important.

Our program is to infer a model of the light distribution in a galaxy from $I$-band photometry. The mass distribution is then parametrized by an $M / L$ for each component and a central density and core radius for a presumed dark halo. Choosing these parameters and the pattern speed of the bar specifies a model. For each of a variety of models we run a hydrodynamical simulation, and reproject the simulation velocities to compare to the Fabry-Perot observations. Comparing models with a range of assumed parameters will constrain the $M / L$ and pattern speed. Here we present preliminary results on the pattern speed of the bar in NGC 4123.

\section{Modeling}

We fit an exponential disk to the 2-D light distribution of NGC 4123 outside the bar region, and subtract the fitted disk from the image. We then fit a spherical Gaussian to the bulge, which is nearly pointlike, and subtract it. The remaining 
light is attributed to the bar, which we do not wish to fit with a parametric model. We deproject the bar light to face-on, using the inclination $\left(i=49.6^{\circ}\right)$ yielded by the disk fit. This assumes that the bar is thin in the $z$-direction, but frees us from having to invent 3-D models for the bar.

We then calculate the gravitational forces in the plane of the disk by direct integration over the bar light. (We assume a particular form for the $z$ dependence of the density distribution; essentially this just determines a softening length.) In other words, the non-axisymmetric forces are determined directly from the photometry. These forces and those of the disk and bulge are then used as inputs to the hydrodynamical code, a 2-D grid code (van Albada 1985, Athanassoula 1992).

The models are parametrized by the $M / L_{I}$ ratios for the components and the bar pattern speed; we ran approximately 80 models. Each model is run for $0.3 \mathrm{Gyr}$ of simulation time, and then the gas velocities are reprojected into radial velocities for comparison with the observed Fabry-Perot velocity field. The photometric decomposition is less than ideal, because the disk of NGC 4123 is not purely exponential but flattens toward the center (Freeman type II). For this reason the $M / L$ values for the components are preliminary; the overall approach remains valid.

\section{Results}

The Fabry-Perot $\mathrm{H} \alpha$ velocity field shows long, straight shocks on the leading edges of the bar, associated with $\mathrm{H} \alpha$ emission and dust lanes. The shocks are angled with respect to the major axis of the bar, in the sense of trailing pitch angle, as in Figure 1a, and those discussed by Athanassoula (1992). Dust lanes of this geometry are common in strong bars; Prendergast (1962) suggested that they were the loci of shocks, and Athanassoula (1992) compared the shapes of shocks in gas flow in Ferrers-ellipsoid potentials to the shapes of these dust lanes.

Our best fitting models show similar straight shocks (Figure 1a). Examination of the models shows an important systematic behavior of these shocks. When corotation is close to the end of the bar, the shocks are straight but angled with respect to the bar. If the corotation radius is increased, i.e. the bar pattern speed slows, the shocks lengthen somewhat, and for yet slower bars, the shocks are actually ahead of the bar, and are rotated forward in the sense of leading pitch angle. This occurs in a variety of families of models.

This behavior can be understood by considering the motion of the gas in the frame corotating with the bar. Inside corotation, the gas is overtaking the bar; it travels across the bar and past the bottom of the bar potential well before the deceleration of the climb out of the potential well causes the gas to "pile up" and shock. This is why the shock forms on the leading edge of the stellar bar (see Prendergast 1983). If the bar pattern speed is decreased, gas inside corotation overtakes the bar more quickly. It will travel farther out of the bar potential well before the deceleration causes the pile-up and shock. Hence, if the pattern speed is slow enough, the shock can occur ahead of the leading edge, outside the bar (Figure 1b). 
Corotation at $6 \mathrm{kpc}$

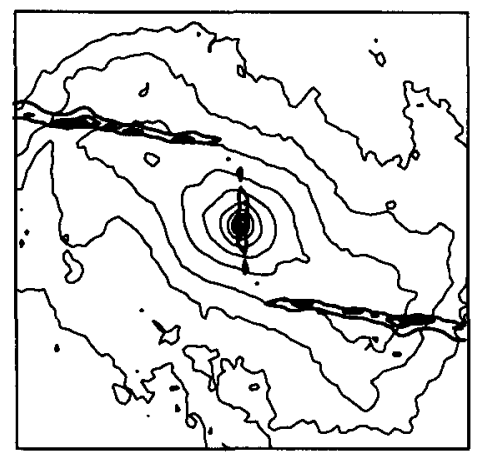

Corotation at $7.5 \mathrm{kpc}$

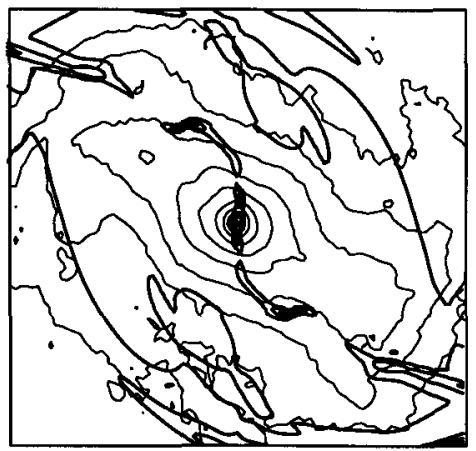

Figure 1. (a) The model which best fits the velocity data, with corotation at $6 \mathrm{kpc}$. The light contours are isophotes of the deprojected $I$-band image, at 0.5 mag intervals. The dark contours outline the high gas density regions, the locations of shocks. The plotted region is 11 kpc by 11 kpc. (b) The same but with corotation at 7.5 kpc.

\section{Conclusions}

The observed velocity field of NGC 4123 shows clear jumps across the dust lanes in the bar, confirming that they are the loci of shocks.

The hydrodynamical models show that flow in a potential derived from a realistic bar shape can reproduce the general features of the observed velocity field, in particular the straight shocks at the leading edge of the bar. Previous simulations (Duval \& Athanassoula 1983, Athanassoula 1992) exhibited these shocks, in bars assumed to be Ferrers ellipsoids.

In simulations with slow-rotating bars, these shocks exist but are rotated forward, so that they actually lead the bar. This disagrees with the observed velocity field, constraining corotation to be at $1.1-1.4$ times the length of the bar semimajor axis. This behavior appears to be generic to many models, and together with the ubiquity of straight dust lanes in strong bars, suggests that strong bars generally have high pattern speeds.

\section{References}

Athanassoula, E. 1992, MNRAS, 259, 345

Duval, M. F. \& Athanassoula, E. 1983, A\&A, 121, 297

Prendergast, K. 1962, unpublished.

Prendergast, K. 1983, in Internal Kinematics and Dynamics of Galaxies, E. Athanassoula, Dordrecht: Reidel, 215

van Albada, G. D. 1985, in The Milky Way Galaxy, H. van Woerden, R. J. Allen, \& W. B. Burton, Dordrecht: Reidel, 547 